\title{
A setup to measure the temperature- dependent heating power of magnetically heated nanoparticles up to high temperature
}

Cite as: Rev. Sci. Instrum. 92, 054905 (2021); https://doi.org/10.1063/5.0038912

Submitted: 27 November 2020 • Accepted: 03 May 2021 • Published Online: 19 May 2021

N. Mille, (D) S. Faure, M. Estrader, et al.

\section{ARTICLES YOU MAY BE INTERESTED IN}

Probing dynamics of nanoparticle chains formation during magnetic hyperthermia using time-dependent high-frequency hysteresis loops

Applied Physics Letters 119, 022407 (2021); https://doi.org/10.1063/5.0056449

Improving energy efficiency of magnetic $\mathrm{CO}_{2}$ methanation by modifying coil design, heating agents, and by using eddy currents as the complementary heating source Journal of Applied Physics 129, 044901 (2021); https://doi.org/10.1063/5.0035655

Simple models for dynamic hysteresis loop calculations of magnetic single-domain nanoparticles: Application to magnetic hyperthermia optimization

Journal of Applied Physics 109, 083921 (2011); https://doi.org/10.1063/1.3551582

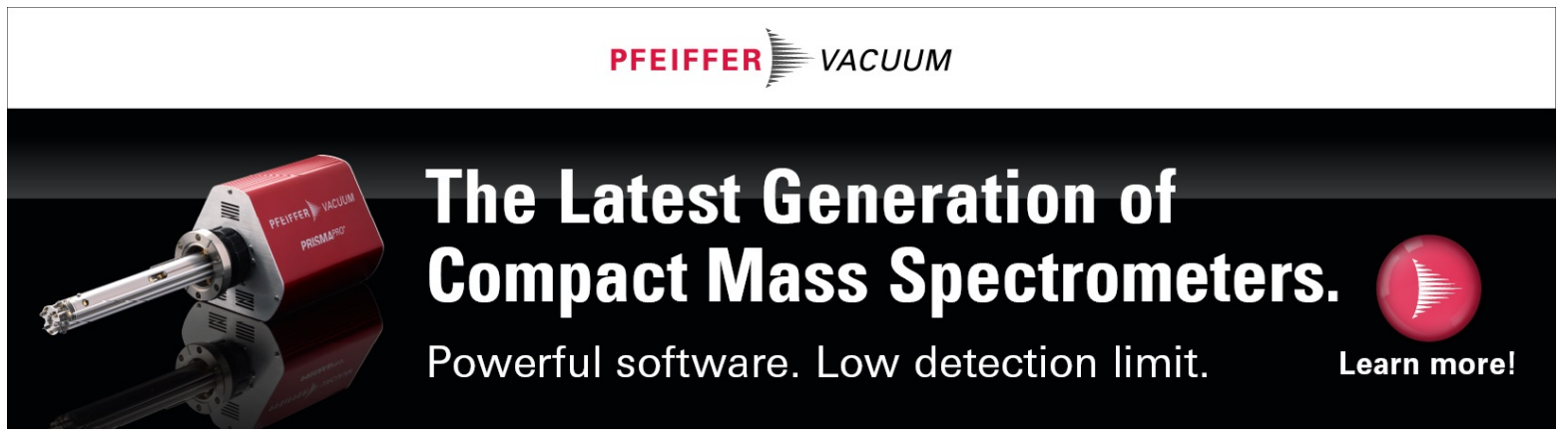




\title{
A setup to measure the temperature-dependent heating power of magnetically heated nanoparticles up to high temperature
}

\author{
Cite as: Rev. Sci. Instrum. 92, 054905 (2021); doi: 10.1063/5.0038912 \\ Submitted: 27 November 2020 - Accepted: 3 May 2021 • \\ Published Online: 19 May 2021
}

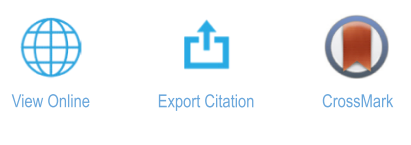

N. Mille, ${ }^{7}$ S. Faure, ${ }^{1}$ (D) M. Estrader, ${ }^{1, a)}$ D. Yi, ${ }^{1}$ J. Marbaix, ${ }^{1}$ D. De Masi, ${ }^{1}$ K. Soulantica, ${ }^{1}$ (D) A. Millán, ${ }^{2}$ B. Chaudret, and J. Carrey ${ }^{1, b)}$ (iD

\author{
AFFILIATIONS \\ ${ }^{1}$ Laboratoire de Physique et Chimie des Nano-Objets (LPNCO), UMR 5215 Université de Toulouse-INSA-CNRS-UPS, \\ 135 av. de Rangueil, 31077 Toulouse Cedex, France \\ ${ }^{2}$ Instituto de Ciencia de Materiales de Aragón, Facultad de Ciencias, C/ Pedro Cerbuna 12, 50009 Zaragoza, Spain
}

\begin{abstract}
a) Present address: Departament de Química Inorgànica i Orgànica, Universitat de Barcelona, Diagonal 645, 08028 Barcelona, Spain and Institute of Nanoscience and Nanotechnology of the University of Barcelona (IN2UB), Barcelona, Spain.

${ }^{b)}$ Author to whom correspondence should be addressed: julian.carrey@insa-toulouse.fr
\end{abstract}

\begin{abstract}
Magnetic heating, namely, the use of heat released by magnetic nanoparticles (MNPs) excited with a high-frequency magnetic field, has so far been mainly used for biological applications. More recently, it has been shown that this heat can be used to catalyze chemical reactions, some of them occurring at temperatures up to $700{ }^{\circ} \mathrm{C}$. The full exploitation of MNP heating properties requires the knowledge of the temperature dependence of their heating power up to high temperatures. Here, a setup to perform such measurements is described based on the use of a pyrometer for high-temperature measurements and on a protocol based on the acquisition of cooling curves, which allows us to take into account calorimeter losses. We demonstrate that the setup permits to perform measurements under a controlled atmosphere on solid state samples up to $550^{\circ} \mathrm{C}$. It should in principle be able to perform measurements up to $900^{\circ} \mathrm{C}$. The method, uncertainties, and possible artifacts are described and analyzed in detail. The influence on losses of putting under vacuum different parts of the calorimeter is measured. To illustrate the setup possibilities, the temperature dependence of heating power is measured on four samples displaying very different behaviors. Their heating power increases or decreases with temperature, displaying temperature sensibilities ranging from -2.5 to $+4.4 \% \mathrm{~K}^{-1}$. This setup is useful to characterize the MNPs for magnetically heated catalysis applications and to produce data that will be used to test models permitting to predict the temperature dependence of MNP heating power.
\end{abstract}

Published under license by AIP Publishing. https://doi.org/10.1063/5.0038912

\section{INTRODUCTION}

The magnetic heating of nanoparticles (NPs) by a highfrequency magnetic field has been widely used in the last few decades, mostly as a cancer treatment, the so-called magnetic hyperthermia. ${ }^{1}$ In 2008, Ceylan et al. proposed a new application of this phenomenon: the activation of a chemical reaction. ${ }^{2}$ The magnetic heating of chemical reactions had already been studied with a first patent dating from $1963^{3}$ but, at the time, without making use of NPs. Ceylan et al. took advantage of the NP nanometric size and of their ability to deliver heat in order to perform several chemical reactions in a microfluidic system. ${ }^{2,4-6}$ This new application of NP magnetic heating has, since then, been studied by several groups, including ours. ${ }^{7-14}$ One should note that the temperatures required for chemical reactions are much higher than the ones used for hyperthermia treatment. Indeed, most studied reactions occurred between 150 and $650^{\circ} \mathrm{C}$, whereas hyperthermia target temperatures range from 40 to $45^{\circ} \mathrm{C}$.

The heating mechanism and the maximum temperature reached by MNPs depend on several parameters, such as their magnetic and structural properties, as well as the heat losses of the system in which they operate. Since NP magnetic properties are temperature dependent and as possible sintering or a phase change can occur at high temperatures, one expects the NP heating power to evolve 
with temperature $T$. The ability of NPs to heat is characterized by their "specific absorption rate" (SAR), which is their heating power per unit mass. It is possible to measure the SAR using two different methods: calorimetric measurement and magnetometric measurement. The calorimetric method consists in measuring the temperature rise in a sample under a high-frequency magnetic field in order to deduce its heating power. The magnetometric one deduces the heating power from high-frequency hysteresis loops since the heating power is directly proportional to area. Both have been used to measure the $\operatorname{SAR}(\mathrm{T})$, i.e., the temperature dependence of the NP heating power.

To our knowledge, the first experimental study of the SAR(T) of MNPs was reported by Veverka et al. ${ }^{15}$ They used the magnetometric method to measure the properties of cobalt ferrite samples but did not provide many details on the setup and the method used. The SAR measured at a field amplitude $\mu_{0} H_{\max }=35 \mathrm{mT}$ and frequency $f=50 \mathrm{kHz}$ increased with temperature from 27 to $80^{\circ} \mathrm{C}$. This was interpreted as resulting from the reduction of the NP anisotropy. Garaio et al. ${ }^{16}$ used the same method on four iron oxide samples under varying field conditions (amplitude and frequency) for temperatures ranging from 10 to $60^{\circ} \mathrm{C}$. They observed for field amplitudes larger than $\mu_{0} H_{\max }=18.8 \mathrm{mT}$ a decrease in SAR with temperature whatever the frequency $(75-1030 \mathrm{kHz})$. These two examples show that different samples can show opposite behaviors.

Calorimetric measurements have also been performed on various types of samples. Since the sample produces heat during the experiment, the deduction of $\operatorname{SAR}(\mathrm{T})$ is less obvious than in the magnetometric measurement. Thanks to an adiabatic setup, Natividad et al. ${ }^{17}$ performed such measurements on magnetite samples for temperatures in the range $120-320 \mathrm{~K}$. They measured the increase in temperature from $T_{i}$ to $T_{i+1}$ when applying the external field during a certain time $t$ and then associated the SAR deduced from this increase to the mean temperature $\left(T_{i}+T_{i+1}\right) / 2$. The heating power of the sample itself allowed them to perform this measurement at different temperatures. The SAR(T) measured on their sample presented a bell shape. They pointed out an important characteristic of the calorimetric method: the requirement to know accurately the heat capacity of the sample, which varies with temperature. In a recent publication, the same group exploited in a clever way an external heating system initially used to guarantee the adiabaticity of their setup to suppress the constraint in knowing the temperature dependence of heat capacities. ${ }^{18}$ Another calorimetric method has been used by Coïsson et al. ${ }^{19}$ on a non-adiabatic setup: they measured the heating curve and the cooling curves for temperatures ranging from 25 to $50{ }^{\circ} \mathrm{C}$ and fitted the heating regime with a theoretical function considered to model the SAR(T). Their method assumed an already known behavior of the SAR with temperature, which is very unlikely in practice.

Most of these studies were performed at rather low temperatures (up to $80^{\circ} \mathrm{C}$ ) since they were made in the scope of magnetic hyperthermia studies. The use of NPs for catalysis implies studying their behavior up to temperatures as high as possible since different catalytic processes are activated at different temperatures. Some experiments have been performed with this in mind. Vinum et al. ${ }^{11}$ performed magnetometric measurements up to $950^{\circ} \mathrm{C}$ on CoNi NPs. This was performed using a vibrating sample magnetometer (VSM) apparatus so that the quasi-static hysteresis loops were measured. In other words, the hysteresis loop was measured at a frequency of the order of $1 \mathrm{mHz}$ since the typical measurement time of a hysteresis loop in a VSM is $10 \mathrm{~min}$. With the NP magnetic properties varying with frequency, this type of measurement is not necessarily representative of the high-frequency behavior. Varsano et al. ${ }^{20}$ published a calorimetric heating power measurement up to $850{ }^{\circ} \mathrm{C}$ of a micrometric $\mathrm{CoNi}$ powder. To do so, they recorded the temperature rise during the heating period and the temperature decrease during the cooling period, thanks to the pyrometer. They computed the SAR by subtracting the slope of the cooling curve from the slope of the heating curve, multiplied by the temperaturedependent heat capacity of their sample. Their work was however focused on the catalytic properties of their sample, and only few details were provided on the measurement method itself and on the setup.

We present here a setup and a method to probe the temperature dependence of NPs up to high temperatures. This method has already been used to characterize $\mathrm{Fe}_{\mathrm{x}} \mathrm{Co}_{1-\mathrm{x}}$ samples for catalysis applications. ${ }^{14}$ We provide here all the technical details of this setup, which is suitable for measurements under controlled atmosphere up to $900^{\circ} \mathrm{C}$. After a presentation of the setup, its limitations and artifacts, and the analysis method, we illustrate its capacity by presenting several results obtained on very different samples, confirming the importance of such a study for a better understanding of the heating behavior of NPs in magnetically heated catalysis.

\section{SETUP AND METHOD}

\section{A. Setup design}

The setup consists of two main parts: a calorimeter and a pyrometer (see Fig. 1). The calorimeter can be made of borosilicate or quartz, quartz being more suitable for very high temperatures, since quartz glassware can be used up to $1050^{\circ} \mathrm{C}$ compared to $500{ }^{\circ} \mathrm{C}$ for the borosilicate one. The one used in this work is made of quartz and was built by a glassblower following our own design (Avitec, France). The calorimeter comprises two compartments, the sample being placed in the inner one. Each compartment is equipped with a valve, which allows gas evacuation through a vacuum line. This allows reducing the heat losses by convection from the sample to its environment. Depending on whether the pyrometer is directly mounted on the calorimeter or not, the inner part can be

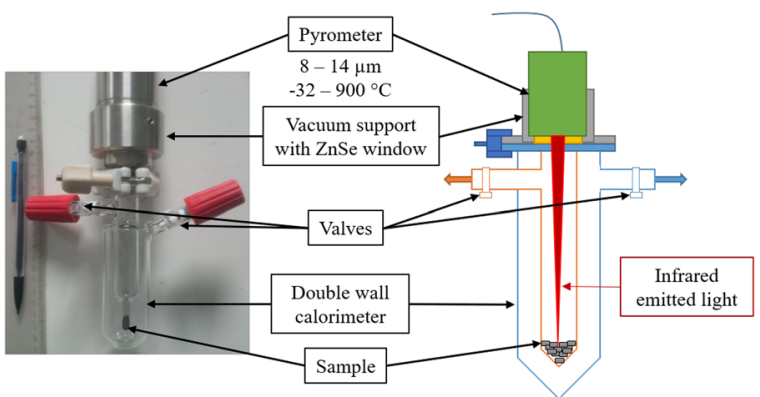

FIG. 1. Photo and scheme of the setup used to measure the temperature dependence of the heating power of NP based samples. 
closed on its upper face by a $\mathrm{ZnSe}$ window enclosed in a support dedicated to fit the pyrometer (LumaSense Vacuum support KF16, Series 5) or by a simple ZnSe window enclosed in a KF16 vacuum flange (Neyco). The calorimeter can be manipulated without the pyrometer: it can for instance be placed in a glove box for the introduction of air sensitive samples. The calorimeter can then be closed with the vacuum support inside the glove box and taken out of the glove box for heating power measurements. This ensures that the atmosphere inside the calorimeter is not contaminated until the end of the experiment. In order to maximize the space under vacuum around the sample, the outer chamber of our calorimeter has been chosen as wide as possible to fit in our magnetic coil, which has a $4 \mathrm{~cm}$ inner diameter. An AC magnetic field is generated by a commercial setup (MP12, Fives Celes, France). It is a standard water-cooled induction oven in which the magnetic field is generated by a two-turn coil made of a hollow copper tube. The magnetic field has a frequency of $300 \mathrm{kHz}$ and a maximum peak amplitude of $\mu_{0} H_{\max }=91.9 \mathrm{mT}$, as calibrated by the manufacturer.

The temperature is measured by a pyrometer (IN-5-Plus, LumaSense Technologies), which converts infrared light into temperature and can be controlled by a computer by a RS- 232 serial port. This pyrometer is calibrated to deduce the temperature from light in the wavelength range of 8-14 $\mu \mathrm{m}$. In this wavelength range, borosilicate and quartz are highly absorbing materials. ${ }^{21,22}$ On the contrary, $\mathrm{ZnSe}$ has a transmission factor of 0.7 , the fully transparent case being 1. Therefore, $\mathrm{ZnSe}$ is most of the time chosen as the window material for the pyrometric measurement in this wavelength range. The emissivity of the sample has to be provided to the pyrometer before the measurement. The pyrometer has been tested with two different lenses supplied by the constructor displaying, respectively, focal lengths of 120 and $300 \mathrm{~mm}$. The height of the pyrometer must be adjusted to have its focal point on the surface of the sample. With the short focal length, the pyrometer is placed directly inside the vacuum support (as illustrated by Fig. 1), which ensures a reproducible and good optical alignment between the sample and the pyrometer, but imposes the position of the focal point inside the calorimeter. Thus, the height of the sample in the calorimeter must be the same for each experiment in order to coincide approximately with the focal point of the pyrometer. However, one could be interested in measuring different volumes of the sample in different calorimeters. With this aim in mind, it is possible to use a pyrometer with a longer focal length $(300 \mathrm{~mm})$ and a $\mathrm{ZnSe}$ window without the dedicated support that fits the pyrometer. When using this configuration, the pyrometer is not connected to the calorimeter anymore. Thus, two supports are needed: one for the pyrometer and one for the calorimeter, which forces the user to fulfill the optical alignment between them for each experiment. This solution permits to measure different quantities of samples and also permits to measure easily the temperature inside different kinds of calorimeters or reactors, provided that they are compatible with the $\mathrm{ZnSe}$ window. For this work, the lens with $300 \mathrm{~mm}$ focal length was chosen. Finally, homemade software in Python language has been written for data acquisition and treatment.

\section{B. Measurement method and limits}

In this section, the protocol, artifacts, and the influence of vacuum on the measurements are presented. To describe them, we use a sample of Naturally Expanded Graphite (NEG), which is extensively described in Sec. III A 1.

\section{Method}

The measurement method is a calorimetric one. The heating power is deduced from the temperature rise in the sample under field. The measurement procedure-illustrated in Fig. 2-is the following: (i) ambient temperature measurement (black straight line); (ii) field application, during which temperature increases (red dashed curve); and (iii) field is switched off and the temperature is recorded until it is back to room temperature (blue dotted curve). It can be noted that the maximum temperature reached during such an experiment depends on the sample and on the field (the more the sample heats, the higher is the maximum temperature).

The data treatment to retrieve the temperature dependence of the heating power is based on the heat transfer equation. When applied to our sample, it gives

$$
\rho V C_{p} \frac{d T}{d t}=P_{e m}-P_{c o n d}-P_{c o n v}-P_{\text {rad }},
$$

with $\rho$ being the volumetric mass density of the entire sample (in $\mathrm{kg} \mathrm{m}^{-3}$ ), $V$ being its volume (in $\mathrm{m}^{3}$ ), $C_{p}$ being its mass heat capacity (in $\mathrm{J} \mathrm{kg}^{-1} \mathrm{~K}^{-1}$ ), $T$ being its temperature (in $\mathrm{K}$ ), $t$ being the time (in s), and $P_{i}$ being the different power contribution of the heat transfer. $P_{e m}$ corresponds to the heat power brought to the sample by electromagnetic heating, which is what one wants to determine. $P_{c o n d}$, $P_{c o n v}$, and $P_{\text {rad }}$ correspond to the heat transfer from the sample to its environment via conduction, convection, and radiation, respectively. When the field is off, $P_{e m}$ is zero. Thus, by subtracting Eq. (1) when the field is off to the same equation when the field is on, and by considering that the heat transfer via conduction, convection, and radiation from the sample to its surrounding is the same for each

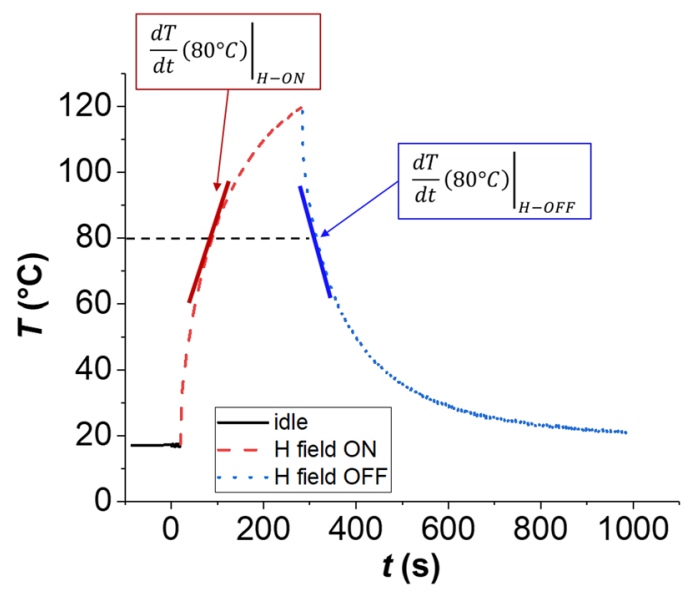

FIG. 2. Typical temperature measurement of the heating of a sample under a high-frequency magnetic field and the cooling after the field is turned off. Data are separated in three parts: (i) The external magnetic field has not yet been turned on (black plain curve). (ii) The field is turned on and the sample heats (red dashed curve). (iii) The field is turned off and the sample cools down (blue dotted curve). The time derivative of the temperature at $T=80^{\circ} \mathrm{C}$ of the heating curve (red plain straight line) and of the cooling curve (blue plain straight line) are illustrated. 
temperature, the subtraction gives

$$
\rho V C_{p}(T)\left(\left.\frac{d T}{d t}(T)\right|_{H-O N}-\left.\frac{d T}{d t}(T)\right|_{H-O F F}\right)=P_{e m}(T) .
$$

As the sample is made of several components (NPs but also potentially ligands and matrices), the prefactor reads

$$
\rho V C_{p}(T)=\sum_{i} m_{i} C_{p, i}(T),
$$

with $m_{i}$ being the mass of element $i$ and $C_{p, i}(T)$ being its heat capacity, which varies with temperature. The SAR of the NPs is most of the time expressed in terms of heating power per unit mass. The final equation used to calculate the SAR(T) from raw data is thus the following:

$$
S A R(T)=\frac{\sum_{i} m_{i} C_{p, i}(T)}{m_{\text {mag }}}\left(\left.\frac{d T}{d t}(T)\right|_{H-O N}-\left.\frac{d T}{d t}(T)\right|_{H-O F F}\right),
$$

with $m_{\text {mag }}$ being the mass of the magnetic material.

The concrete implementation of the method is made via python scripts in the following way: (i) Data acquisition of the complete curve (heating and cooling curves) with a time step of $0.5 \mathrm{~s}$, as exemplified in Fig. 2. (ii) Numerical smoothing of the curve with potentially variable parameters. The method used is a savgol filter already implemented in Python, with time windows from 1 to $3 \mathrm{~s}$ and typical polynomial order between 5 and 9. (iii) Time derivation of the heating and cooling curves made with the gradient function of the numpy library in Python (see the documentation for more details). ${ }^{23}$ (iv) Interpolation of the two derivative curves to reconstruct them with a given temperature step. (v) Subtraction of the cooling derivative curve to the heating derivative curve for each temperature (which is why the previous step is mandatory). (vi) Multiplication of the result by the heat capacity and mass prefactor [see Eq. (4)].

\section{Limits and uncertainty}

a. Uncertainty on temperature measurements and on the deduced SAR. According to the specification of the pyrometer, ${ }^{24}$ the temperature is known with an accuracy of $\left.\Delta T\right|_{p y r o}= \pm 1$. Several other errors can arise on the temperature measurement. First, the temperature at the sample surface is lower than the mean temperature of the whole sample since radiative and convective losses originate from the surface. The temperature measured here is thus slightly underestimated, as well as the deduced SAR. We have not attempted to quantify and correct this error. Second, the infrared measurement implies a knowledge of the material emissivity. It is hard both theoretically and in practice to know this parameter and its temperature dependence. The best way would be to calibrate the emissivity for each sample: the sample temperature should be measured with the pyrometer using an oven in which the temperature would be accurately known by an independent method; for several steps of temperature, the emissivity of the sample would be modified until the two values of temperature match. This would permit to obtain the emissivity value as a function of the temperature. However, this is very time consuming and can degrade the sample before measuring it under a high-frequency magnetic field. We have thus used a simpler method: the samples were let into our magnetic coil for $1 \mathrm{~h}$ until they reach an equilibrium temperature. The latter is known and reproducible due to the fact that the coil is cooled by a flow of water at $18.5^{\circ}$; the emissivity of the samples was then deduced at this temperature, and the temperature dependence of its emissivity was neglected. It should be noted that this dependence is rather weak. For example, the emissivity of graphite varies from 0.7 to 0.8 in the temperature range $0-3600{ }^{\circ} \mathrm{C}$. The emissivity of cobalt varies from 0.13 to 0.23 between 500 and $1000^{\circ} \mathrm{C}$ and that of iron oxides varies from 0.85 to 0.89 between 500 and $1200{ }^{\circ} \mathrm{C} .{ }^{25}$ We consider that the emissivity of the material, which is in the range [0-1], is estimated at \pm 0.05 with this technique. In our temperature range, changing the emissivity parameter of \pm 0.05 leads to a change in temperature of $\left.\Delta T\right|_{e m i}= \pm 1^{\circ} \mathrm{C}$. We have thus two quantifiable error sources on the temperature measurement (the one given by the pyrometer itself and the one arising from emissivity), which are uncorrelated. When such ranges are known without more information, the range divided by the square root of three is an upper limit of the uncertainty. ${ }^{26}$ Then, using the classical uncertainty combination formula in the case of uncorrelated error sources, the final uncertainty on the temperature measurement is given by

$$
\left.u(T)\right|_{\text {meas }}=\left(\left(1^{\circ} \mathrm{C} / \sqrt{3}\right)^{2}+\left(1^{\circ} \mathrm{C} / \sqrt{3}\right)^{2}\right)^{1 / 2}=0.8^{\circ} \mathrm{C} .
$$

To deduce the SAR value of the sample, we are interested in a temperature difference. Any systematic error on the temperature is thus canceled. Only the variability of the temperature measurement is relevant in this case. We have deduced the latter via the statistical method on temperature measurements. To do so, we have heated a piece of Naturally Expanded Graphite (NEG) at different thermal equilibria, thanks to different applied fields. We have acquired between 20 and 40 temperature measurements for each equilibrium and calculated the variability of the measurements. The highest uncertainty is at low temperature, and we have decided to use this one for the whole temperature range as a high estimation. The resulting uncertainty, here the square root of the variance of the measurements, ${ }^{26}$ is $\left.u(T)\right|_{\text {var }}=0.07^{\circ} \mathrm{C}$.

The sample masses were measured with a quartz balance with microgram precision, and the heat capacities were obtained from the literature. ${ }^{27,28}$ Hence, we assume that the heat capacities and masses of the different elements are known with sufficient accuracy to consider the temperature measurement as the main source of error. Starting with Eq. (4), we have used the propagation law of uncertainties, considering first the variability of the temperature as the only source of error and then that the numerical derivative of the temperature is equivalent to a subtraction of two temperatures divided by the time step $d t$. The error on the derivative difference is thus four times the error on the temperature, and the uncertainty on the $\operatorname{SAR}(\mathrm{T})$ is given by

$$
u(S A R)=\sqrt{\left(\frac{\sum_{i} m_{i} C_{p, i}(T)}{m_{\text {mag }}}\right)^{2} \times 4 \times \frac{\left.u^{2}(T)\right|_{\text {var }}}{d t}} .
$$

b. Transient regime. Concerning the model used to treat the data, we have made the approximation that the heat transfer at a specific temperature during the heating period is the same as during the cooling period at the same temperature. This approximation needs to be discussed. The following discussion is qualitative 
and more experimental data are needed to verify it, such as temperature measurements simultaneously on different parts of the calorimeters.

Let us consider the heat transfer via conduction from the sample to the surrounding glass. Figure 3 shows an example of raw temperature measurements where the data points that will be discussed below are marked. At a very short time after starting the experiment, when the temperature is close to the ambient one and the field is on (point A in Fig. 3), the sample heats very fast, faster than the glass, so these two elements are not at the same temperature. Long after the field has been switched off, near the end of the experiment (point D in Fig. 3), the temperature of the sample and of the glass are expected to be similar and close to room temperature. The thermal losses are thus different for those two temperatures. However, we subtract the slope of the second to the slope of the first assuming that the losses are the same in both cases. This reasoning is also valid when considering two data points at high temperatures: when the sample has been heated near high-temperature thermal equilibrium (point B in Fig. 3), the glass and the sample have similar temperatures. When the field is shut off (point $\mathrm{C}$ in Fig. 3), the glass temperature decreases faster than that of the sample, leading to a temperature gradient between the two. However, we subtract the slope at point $C$ to the slope at point $\mathrm{B}$ assuming that the losses are the same. This reasoning is also true for the convection process and for the gradient occurring between the reactor glass and the ambient atmosphere. Thus, we suffer from a lack of quantification of those heat transfer parameters, which vary for each sample, with the temperature range and the time scale.

Another phenomenon has been observed when the field has just been applied or shut down. We illustrate it with the sample of Natural Expanded Graphite (NGE). In Fig. 4, the time derivative of the temperature cooling curve is plotted, i.e., when the sample has been heated and the field is turned off, for three different applied field intensities. If those curves are multiplied by the heat capacity prefactor, the heat transfer from the sample to its environment is obtained [e.g., glass and outer air; see Eq. (1) with $P_{e m}=0$ ]. The heat

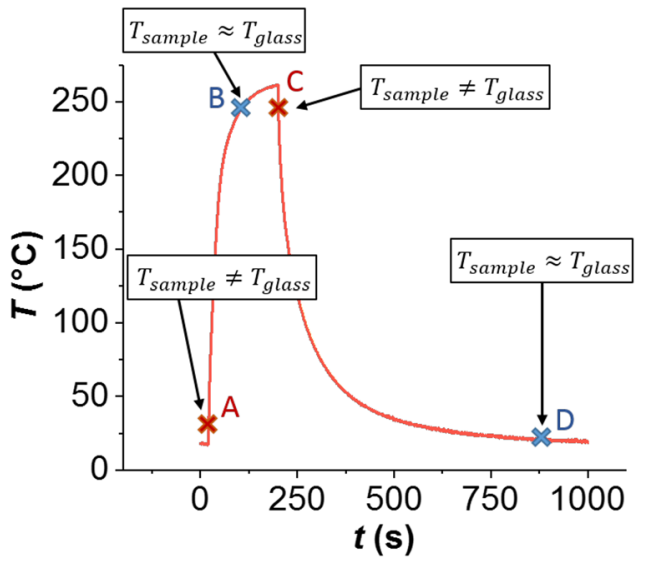

FIG. 3. Typical raw temperature measurement curve. The points $A, B, C$, and $D$ show different times of interest of the heating and cooling curves (see the text for details).

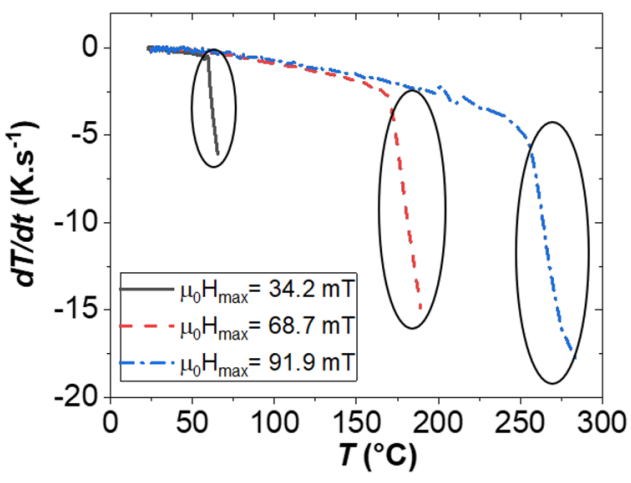

FIG. 4. Time derivative of the temperature as a function of temperature after turning off the field for three different field amplitudes. Measurements were taken on a sample of naturally expensed graphite (NGE) measured at atmospheric pressure.

transfer from the sample to its environment at a specific temperature is not supposed to change with the previously applied field. Only the maximum temperature reached is higher when a higher field is applied (see Fig. 4). The losses, as expected, overlap well. However, for the three regions highlighted by black circles, this is not the case. Those regions correspond to the time after switching off the field, close to the maximum temperature reached during each experiment. In the example shown in Fig. 4, the time period during which the curves do not overlap lasts $4 \mathrm{~s}$ after turning off the field. This phenomenon is also observed with the same temporality directly after turning on the external field. Thus, we do not take into account this short time (few seconds) after turning on or off the field in our data treatment.

c. Influence of vacuum. Thanks to the valves, we are able to put under vacuum one or both parts of the calorimeter. The purpose is to reduce the heat transfer via convection from the sample to the gas present in the calorimeter or to the atmosphere. To study the influence of this parameter on our measurements, we have performed three experiments on the same NGE sample both in the presence and in the absence of air in the different chambers of the calorimeter.

The results are shown in Fig. 5 for three different configurations: atmospheric pressure in both chambers, vacuum in the outer chamber, and vacuum in both chambers (see Fig. 1 for the position of the chambers). In Fig. 5(a), raw temperature data are depicted. Figures 5(b) and 5(c) show the time derivative of the temperature as a function of the temperature for the three configurations when applying the field and after turning off the field. Evacuating the outer chamber has a significant influence only above $\sim 100^{\circ} \mathrm{C}$ [see the red and blue curves in Fig. 5(c)]. Vacuum in the inner chamber does not produce any modification in the heat losses experienced by the sample [see the overlap between the blue and green curves in Fig. 5(c)]. This indicates that the heat transfer via convection from the sample to the gas present in the inner chamber is not the main mechanism of losses, at least at those temperatures. More likely, heat losses seem to occur via conduction from the sample to the reactor glass and then via convection from the reactor glass to the environment. 

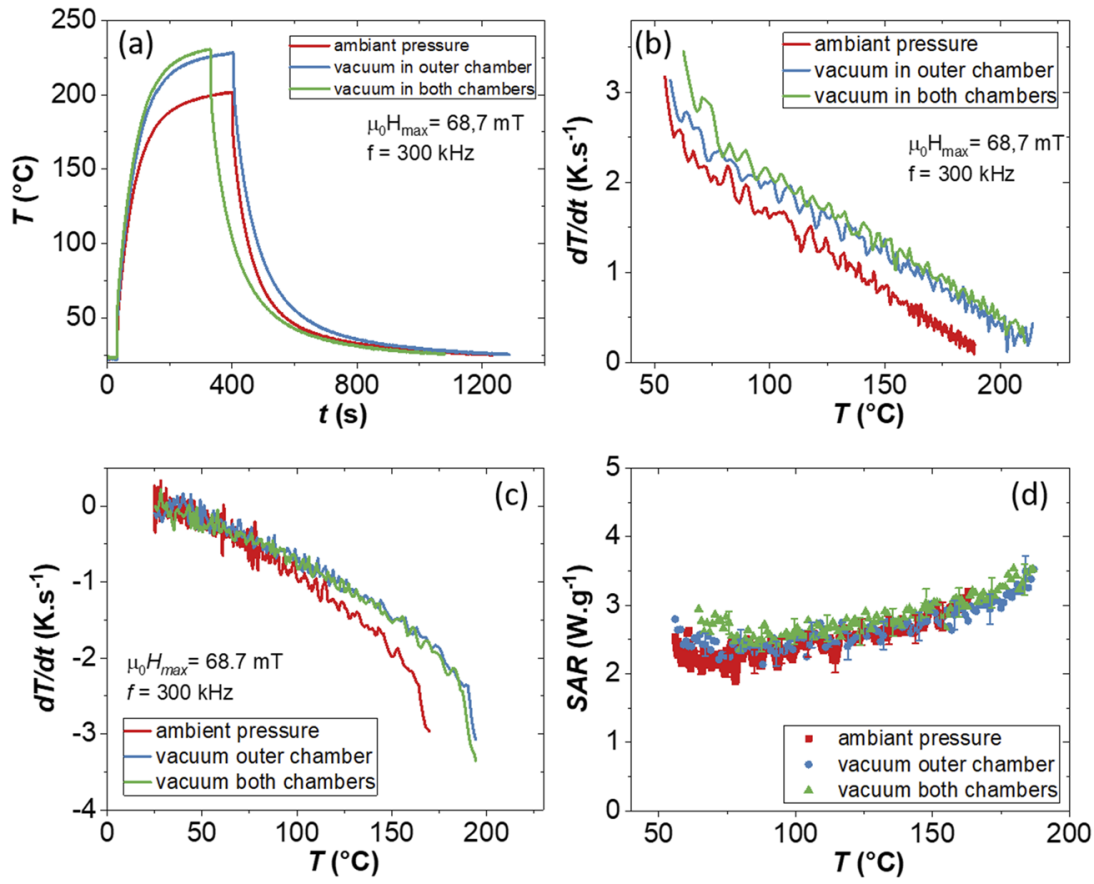

FIG. 5. (a) Raw temperature measurement on a sample of NGE with an applied field of intensity $\mu_{0} H_{\max }=68.7 \mathrm{mT}$ and frequency $f=300 \mathrm{kHz}$ for three configurations: ambient pressure in both compartments (red curve), vacuum in the outer chamber of the calorimeter (blue curve), and vacuum in both chambers of the calorimeter (green curve). (b) Time derivative of the temperature as a function of temperature just after the field has been turned on. (c) Time derivative of the temperature as a function of the temperature after turning off the field. (d) SAR(T) deduced from the measurements.
In Fig. 5(d), the SAR is plotted as a function of the temperature for the three different configurations. In principle, the SAR(T) curves are supposed to be the same whatever the heat losses since our data treatment is supposed to eliminate the influence of losses on the final result. The main difference between the three cases is the maximum temperature reached, which is $165^{\circ} \mathrm{C}$ at ambient pressure and $186^{\circ} \mathrm{C}$ when both chambers are under vacuum. The three curves match well, but a slight difference between the three curves can be observed: the SAR at ambient pressure is slightly lower than the SAR obtained under vacuum in the outer chamber, which is slightly lower than the SAR with vacuum in both chambers. This gives us a hint on the error made when it is assumed that the heat losses from the sample to its surrounding are equal for the same temperature between the heating and cooling period. The difference is $0.5 \mathrm{~W} \mathrm{~g}^{-1}$ in this case, larger than the calculated source of the uncertainty for the SAR, estimated with Eq. (6) at $0.1 \mathrm{~W} \mathrm{~g}^{-1}$. In the graphs, which will be shown in this paper, the error bars on temperature are calculated with Eq. (5) and the error bars on the SAR are calculated according to Eq. (6). This uncertainty is underestimated because of the other sources of error detailed above.

\section{SETUP POSSIBILITIES: EXPERIMENTAL STUDY}

We present here the experimental results aiming at illustrating the possibilities of this setup and of our analysis protocol. Measurements on four different samples, expected to behave very differently, were performed. Figure 6 presents images of three samples used in this paper (NEG, $\mathrm{Fe}_{3} \mathrm{O}_{4} \mathrm{MNPs}$, and cobalt nanorods) and basic magnetic characterization of two of them. The fourth sample, FeCo nanoparticles, is extensively characterized in Ref. 14 and is not shown in this figure.

\section{A. Naturally expanded graphite: A non-magnetic conducting sample \\ 1. Sample description}

NEG (naturally expanded graphite) is a common material used mostly as a heat insulator at high temperatures or as a primary material to obtain graphite foils. It is obtained from natural graphite treated by nitric acid and sulfuric acid. ${ }^{29}$ It is a diamagnetic material and a thermal and electrical conducting material. ${ }^{29}$ Our samples were prepared from a circular piece of NGE $(4 \mathrm{~cm}$ diameter and $0.5 \mathrm{~cm}$ thick; see Fig. 6(a)]. The piece was cut along its diameter in order to measure its resistivity. Then, a $0.5 \mathrm{~cm}$ cubic sample was cut in order to perform SAR measurements in our calorimeter.

As a diamagnetic conducting material, NEG heating under an alternating magnetic field is only due to eddy currents, which depend on the material conductivity. The conductivity of NEG is anisotropic and varies depending on the preparation, and very few data concerning its temperature dependence can be found. Hence, we measured it using a hot plate and our pyrometer in the range $30-165^{\circ} \mathrm{C}$. The four-point resistivity was measured using a multimeter (Keithley 2400) along the $4 \mathrm{~cm}$ length of the sample. The temperature dependence of the resistivity was not significant compared to the variability of our measurement. The mean resistivity in the temperature range $30-165^{\circ} \mathrm{C}$ was $7.2 \times 10^{-4} \Omega \mathrm{m}^{-1}$. We assumed that the heat capacity of our sample and its temperature dependence were the same as those of graphite. ${ }^{30}$

\section{Expected $S A R(T)$}

The temperature dependence of eddy current heating mainly originates from the temperature dependence of the resistivity and strongly depends on whether the electromagnetic power is completely absorbed by the material or not. When the incident wave 

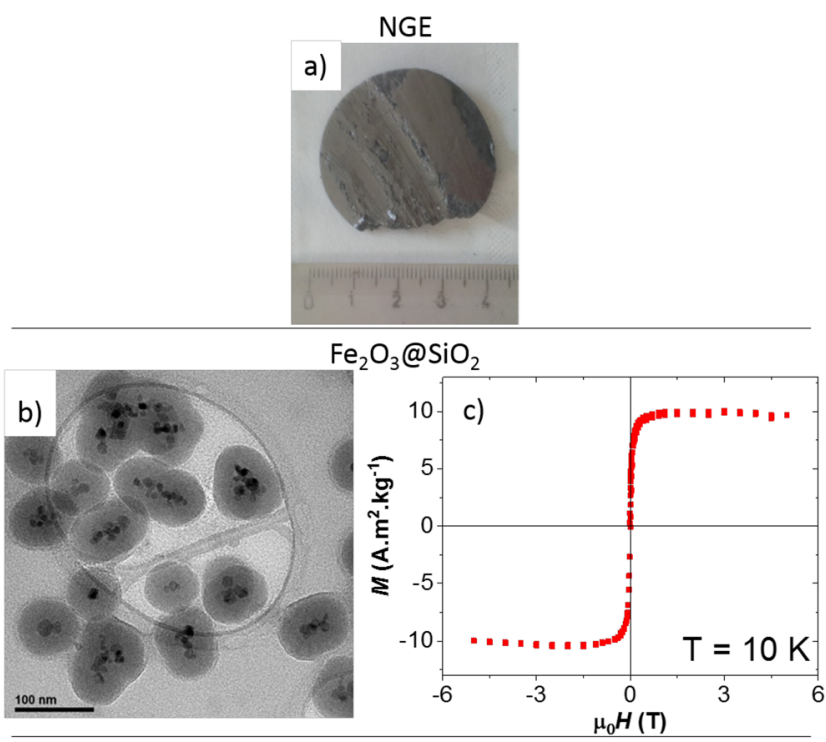

Co nanorods on Siralox
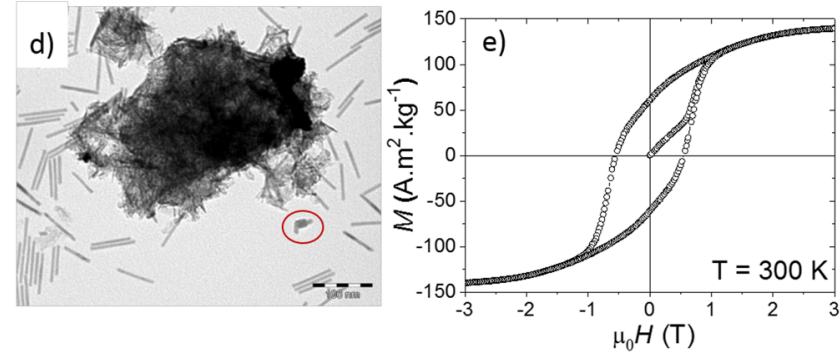

FIG. 6. Samples used as examples to show the capability of the setup: (a) NEG raw piece. (b) TEM image of the $\mathrm{Fe}_{2} \mathrm{O}_{3} @ \mathrm{SiO}_{2} \mathrm{NPs}$; the scale bar is $100 \mathrm{~nm}$ wide. (c) SQUID measurements at $T=10 \mathrm{~K}$. (d) TEM image of cobalt nanorods supported on Siralox-5@; a residual non-rod-shaped particle is highlighted by a red circle. (e) VSM measurements performed at $T=300 \mathrm{~K}$.

is weakly absorbed (weak skin effect), the heating power decreases with resistivity. ${ }^{31}$ However, if the wave is fully absorbed (strong skin effect), it is rather constant since all the energy is transferred to the absorbing material; only the thickness on which it is absorbed varies. ${ }^{32}$ Here, in the case of NEG, considering an applied field of frequency $f=300 \mathrm{kHz}$, the previously measured resistivity, and the basic formula of penetration depth, ${ }^{32}$ the penetration depth is $28 \mu \mathrm{m}$. Our sample being in the millimeter range, the whole incident wave is thus absorbed. Moreover, this material is a good thermal conductor, ${ }^{29}$ which reduces the potential temperature inhomogeneity. This means that the regime is the one where the energy is fully absorbed, and the heating power is expected to be temperature independent.

\section{3. $S A R(T)$ results}

We measured the evolution of the sample temperature when it was submitted to three different field intensities $\left(\mu_{0} H_{\max }\right.$ $=34.2,68.7$, and $91.9 \mathrm{mT}$ ) at a field frequency of $300 \mathrm{kHz}$, following the protocol detailed in Sec. II B 1. The temperature measurements were done under ambient air condition. These raw data were

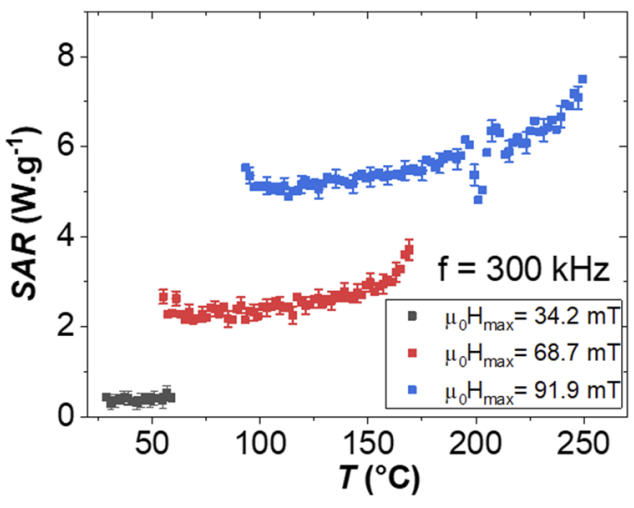

FIG. 7. Heating power as a function of the temperature for a sample of NEG for three different magnetic fields at a frequency $f=300 \mathrm{kHz}$. Since we deduce the first $4 \mathrm{~s}$ of measurement, which brings the material to a higher temperature if a higher field is applied, the starting temperature of the treated data is higher with a higher applied field.

converted into the SAR(T) using $C_{\mathrm{p}}(T)$ curves for graphite from the literature. ${ }^{27}$ Figure 7 shows the SAR variation as a function of temperature obtained with three different field intensities. We observe that, as expected, the higher the field, the higher is the SAR. At $\mu_{0} H_{\max }=34.2 \mathrm{mT}$, the SAR varies between $0.44 \pm 0.1 \mathrm{~W} \mathrm{~g}^{-1}$ at $T=29.5^{\circ} \mathrm{C}$ and $0.58 \pm 0.1 \mathrm{~W} \mathrm{~g}^{-1}$ at $T=57.5^{\circ} \mathrm{C}$. At $\mu_{0} H_{\max }=91.9$ $\mathrm{mT}$, the SAR varies between $5.1 \pm 0.1 \mathrm{~W} \mathrm{~g}^{-1}$ at $T=99^{\circ} \mathrm{C}$ and $7.2 \pm 0.1 \mathrm{~W} \mathrm{~g}^{-1}$ at $T=245^{\circ} \mathrm{C}$. For this highest applied field in the temperature range $T=[99-245]^{\circ} \mathrm{C}$, the mean increase in the SAR per Kelvin in this range is $\Delta(S A R) / \Delta T=+0.014 \mathrm{~W} \mathrm{~g}^{-1} \mathrm{~K}^{-1}$ or $+0.28 \% \mathrm{~K}^{-1}$. This variation is weak, as will become obvious by comparison to that measured on other samples.

This difference, as compared to the expected behavior, can arise from our lack of knowledge concerning $C \mathrm{p}(T)$ of our sample, the variation of which with temperature might be lower than that of raw graphite. This example shows that the setup permits to measure the heat released by a non-magnetic sample and its temperature variation.

\section{B. $\mathrm{Fe}_{2} \mathrm{O}_{3} @ \mathrm{SiO}_{2}$ NPs: Superparamagnetic sample 1. Sample description}

Iron oxide is the most widely used material as NPs for hyperthermia treatment. Our sample is made of clusters of $\sim 6 \gamma-\mathrm{Fe}_{2} \mathrm{O}_{3}$ NPs encapsulated into a silica shell. The iron oxide cores were synthetized by Massart's method, ${ }^{33}$ coated with citric acid and resuspended in water. The silica coating was performed in a $71 \%$ ethanol solution with $0.3 \mathrm{~mol} / \mathrm{l}$ of ammonia by the addition of TEOS and $24 \mathrm{~h}$ stirring in seven consecutive steps. A TEM image of the sample is shown in Fig. 6(b). Each $\gamma-\mathrm{Fe}_{2} \mathrm{O}_{3} \mathrm{NP}$ is spherical and has a diameter of $10.4 \pm 2.1 \mathrm{~nm}$. Dynamic light scattering (DLS) measurements indicate that the hydrodynamic diameter is $115 \pm 11 \mathrm{~nm}$ for the complete $\gamma-\mathrm{Fe}_{2} \mathrm{O}_{3} @ \mathrm{SiO}_{2} \mathrm{NPs}$, in good agreement with the TEM images.

Magnetic characterizations have been performed using a superconductive quantum interference device (SQUID) on $10.6 \mathrm{mg}$ of powder. The magnetization curve at $T=10 \mathrm{~K}$ is shown in Fig. 6(c), 
evidencing that a coercive field at $T=10 \mathrm{~K}$ is $\mu_{0} H_{c}=21 \mathrm{mT}$. The sample used for the SAR measurement consists in $60 \mathrm{mg}$ of powder, composed of $3.9 \mathrm{mg}$ of $\mathrm{Fe}_{2} \mathrm{O}_{3}$ and $56.1 \mathrm{mg}$ of silica.

\section{2. $S A R(T)$ results}

The heating power as a function of the temperature was measured with an applied field of $\mu_{0} H_{\max }=91.9 \mathrm{mT}$ at a frequency of $f=300 \mathrm{kHz}$. The temperature measurements were performed with vacuum in both chambers of the calorimeter. The result is presented in Fig. 8, where Fig. 8(a) is the raw temperature and Figs. 8(b) and 8 (c) are the derivatives of the temperature as a function of the temperature with the field on and off, respectively. The SAR of this sample decreases from $21.1 \pm 1.5 \mathrm{~W} / \mathrm{g}$ at $T=43^{\circ} \mathrm{C}$ to $5.0 \pm 1.5 \mathrm{~W} / \mathrm{g}$ at $T=73{ }^{\circ} \mathrm{C}$. Within this range of $T=[43-73]^{\circ} \mathrm{C}$, the SAR variation is $\triangle S A R / \triangle T=-0.54 \mathrm{~W} \mathrm{~g}^{-1} \mathrm{~K}^{-1}$ or $-2.5 \% \mathrm{~K}^{-1}$, which is significant.

We attribute this decrease to the reduction in the coercive field with temperature. As the coercive field at $T=10 \mathrm{~K}$ is only $\mu_{0} H_{c}=21 \mathrm{mT}$, we infer that it is smaller than our applied field of $\mu_{0} H_{\max }=91.9 \mathrm{mT}$ at our starting temperature $T=43^{\circ} \mathrm{C}$, even for a frequency $f=300 \mathrm{kHz}$. If the coercive field at $300 \mathrm{kHz}$ is already smaller than our applied magnetic field at the starting temperature, its decrease induces a decrease in hysteresis area and thus of the heating power of the sample. ${ }^{34}$

One can see that the maximum temperature reached is only $73{ }^{\circ} \mathrm{C}$, compared to $245^{\circ} \mathrm{C}$ obtained with the NEG sample, even if the SAR values are comparable $\left(\sim 7 \mathrm{~W} \mathrm{~g}^{-1}\right.$ for NEG and $\sim 5 \mathrm{~W} \mathrm{~g} \mathrm{~g}^{-1}$ for the present sample). This is due to the small amount of heating material: only $3.9 \mathrm{mg}$. This example illustrates that, with our setup, measuring at high temperatures requires using a large enough amount of the heating agent. Potentially, samples with a very low heating power cannot be measured at high temperatures.

\section{Cobalt nanorods: Ferromagnetic sample 1. Sample description}

Cobalt nanorods (Co-NRs) are studied as candidates for magnetic recording applications and as building blocks for rare-earth free magnets. ${ }^{35}$ Recently, our laboratory used them combined with other softer NPs as the heating agent for chemical reaction catalysis. ${ }^{36}$ They have a great potential for this application due to the high Curie temperature of cobalt. Indeed, Curie temperature provides an upper limit to the temperature reachable via magnetic heating for a particular material. Their high-temperature heating behavior is thus of double interest: studying them as the heating agent for chemical reactions requiring high temperatures and comparing them with the previously described soft $\mathrm{Fe}_{2} \mathrm{O}_{3} @ \mathrm{SiO}_{2} \mathrm{NPs}$.

The synthesis methods of the cobalt nanorods have been published elsewhere ${ }^{36}$ (see the supplementary material ESI-1.4 in this reference). It is based on the thermal decomposition of a cobalt precursor in the presence of acid and amine ligands. The synthesis is performed at $T=110^{\circ} \mathrm{C}$. It leads to $90 \mathrm{~nm}$ long cobalt nanorods with a diameter of $5 \mathrm{~nm}$, with residual spherical NPs of cobalt. The cobalt nanorods have a monocrystalline hcp structure with their hard axis (c axis of the hcp-shell) along the length of the rod, as previously observed by HRTEM.

Magnetic characterizations have been performed using a vibrating sample magnetometer (VSM). The hysteresis loop at $T$ $=300 \mathrm{~K}$ is shown in Fig. 6(e). This sample is clearly ferromagnetic at room temperature, with a coercive field $\mu_{0} H_{c}(T=300 \mathrm{~K})=553 \mathrm{mT}$, as expected for hcp-cobalt, which has a strong room temperature magnetocrystalline anisotropy. ${ }^{38,39}$ Moreover, in our case, shape and surface anisotropies add to the magnetocrystalline one because of the coincidence between the rod long axis and the $c$ axis of the hcp-shell.
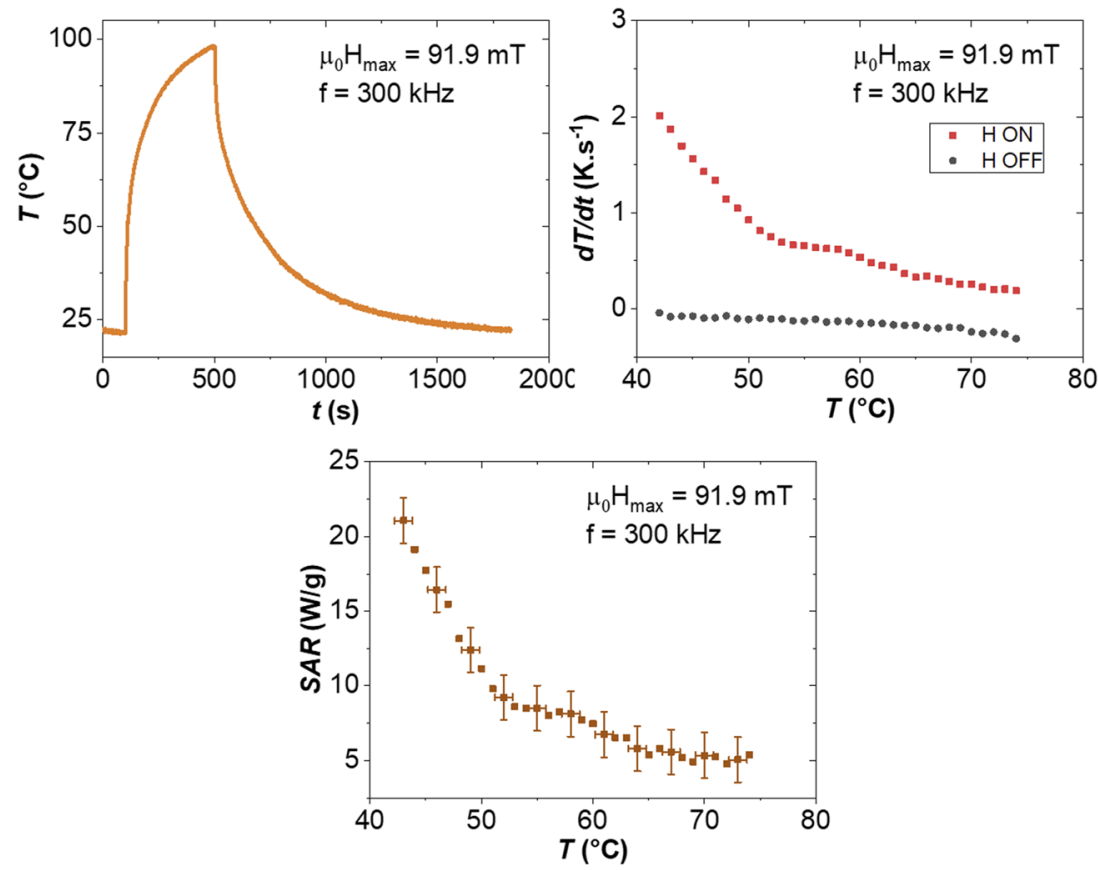

FIG. 8. (a) Raw temperature curve of the $\mathrm{Fe}_{2} \mathrm{O}_{3} @ \mathrm{SiO}_{2}$ sample for a field $\mu_{0} H_{\max }=91.9 \mathrm{mT}$ at frequency $f=300 \mathrm{kHz}$. Both calorimeter chambers were under vacuum. (b) Time derivative of the temperature as a function of temperature for both regimes: when the field is on (red squares) and when the field is shut off after the heating (black dots). (c) SAR as a function of temperature. 
The Co-NRs were supported on Siralox-5๑, composed of mesoporous silica-alumina microparticles. This has been done here for two reasons: (i) to be as close as possible to the catalytic conditions and (ii) to have more material to test with the pyrometer. Indeed, $10 \mathrm{mg}$ of Co-NRs were not enough to fill the $2 \mathrm{~mm}$ wide measurement spot of the pyrometer. The typical samples used were composed of $100 \mathrm{mg}$ of powder (10 mg of Co-NRs and $90 \mathrm{mg}$ of Siralox-5(). A TEM image of the final sample is shown in Fig. 6(d). A residual particle that is not a nanorod has been marked with a red circle.

\section{2. $S A R(T)$ results}

SAR(T) measurements on this sample were performed under the same field conditions as before, i.e., $\mu_{0} H_{\max }=91.9 \mathrm{mT}$ and $f=300 \mathrm{kHz}$, but with argon at ambient pressure in both calorimeter chambers. Raw temperature curves are shown in Fig. 9. In Fig. 9(a), the temperature evolution during the first measurement run is plotted. It can be seen that, at first, temperature increases smoothly from $T=20^{\circ} \mathrm{C}$ at $t=100 \mathrm{~s}$ (field on) to $T=121^{\circ} \mathrm{C}$ at $t=226 \mathrm{~s}$. At this time, a thermal runaway where the temperature sharply increases from $T=121{ }^{\circ} \mathrm{C}$ to $T=260{ }^{\circ} \mathrm{C}$ within only $6 \mathrm{~s}$ is observed. The temperature curve then oscillates randomly before showing another sharp increase in temperature up to $T=324^{\circ} \mathrm{C}$ at $t=248 \mathrm{~s}$ after which the field has been stopped. Figure 9(b) shows the temperature curve of the second and third measurements performed just after the first one. The behavior is not the same. During the second run (blue plain curve), the temperature increases sharply up to more than $300^{\circ} \mathrm{C}$ and oscillates randomly. On the third experiment (green dotted curve), the temperature grows following a classic damped exponential behavior. This complete behavior was observed also for three other measurements (not shown here) on three fresh samples. Each time, the first measurement shows a thermal runaway at approximately the same temperature at the same time, followed by oscillations and irreversible changes of the sample.

We attribute those irreversible changes to a sintering of the cobalt nanorods. They are stable at least up to $T=110^{\circ} \mathrm{C}$, temperature of the synthesis, so that a fast sintering of the NPs occurring at $120^{\circ} \mathrm{C}$ only could appear surprising. However, it should be taken into account that due to heat diffusion inside the sample, the temperature of the NPs themselves might be higher than the mean temperature of the sample. In addition, this sharp increase might also have a purely magnetic origin (decrease in the coercive field) or be a combination of magnetic effects and sintering, the former appearing at low temperature, being followed by sintering at high temperatures.
Previous data are not suitable for our mathematical treatment as they do not only contain the heating behavior of the sample but also its irreversible degradation. To avoid this, we have performed another experiment on a fresh sample stopping the field right before the thermal runaway. The results are shown in Fig. 10. The raw temperature measurements presented in Fig. 10(a) show no thermal runaway. No irreversible changes occurred during this experiment. The time derivative of the temperature as a function of the temperature with and without the field is depicted in Fig. 10(b). The deduced SAR as a function of the temperature is plotted in Fig. 10(c). The SAR increases from $4.9 \pm 1 \mathrm{~W} \mathrm{~g}^{-1}$ at $T=40^{\circ} \mathrm{C}$ to $14.0 \pm 1 \mathrm{~W} \mathrm{~g}^{-1}$ at $T=82^{\circ} \mathrm{C}$. In this temperature range, the variation in SAR is $\triangle S A R / \triangle T=+0.22 \mathrm{~W} \mathrm{~g}^{-1} \mathrm{~K}^{-1}$ or $+4.4 \% \mathrm{~K}^{-1}$.

This increase is attributed to the decrease in coercivity of the sample with temperature. Two processes are involved in this decrease: (i) the very strong reduction in the first order magnetocrystalline anisotropy of hcp-cobalt in this temperature region ${ }^{38,39}$ and (ii) the ease of magnetic reversal via the thermal energy. These effects, combined, give a sharply decreasing coercivity of cobalt nanorods with temperature. At room temperature, our applied field is lower than the coercive field, leading to a small SAR. As the coercive field gets closer to the applied field, the magnetic reversal is facilitated and the hysteresis of sample starts to open, leading to an increase in the heating power. In such a sample, the following behavior is theoretically expected: the more it heats, the higher the temperature, and as the temperature increases, it heats even more. We cannot truthfully attribute the thermal runaway to this "snowbowl effect" since it leads to irreversible changes of the samples, as already mentioned previously.

This example and its comparison with the $\mathrm{Fe}_{2} \mathrm{O}_{3} @ \mathrm{SiO}_{2}$ sample show how interesting are high-temperature heating power measurements. The same effect (reduction in the coercive field with temperature) leads to opposite heating behavior. Our results show that the thermal stability of the sample is mandatory to get reproducible high-temperature behavior but also reveal a side use of this setup: studying the NP's thermal stability, which is also of great interest for chemical reaction catalysis application.

\section{FeCo and chain formation}

We have used this setup to study a series of NPs of different $\mathrm{Fe}_{\mathrm{x}} \mathrm{Co}_{1-\mathrm{x}}$ alloys. The NPs, the size of which varies between 10 and $23 \mathrm{~nm}$, are here again dispersed onto Siralox-5@. These samples have been used to heat different chemical reactions, mostly hydrocarbon
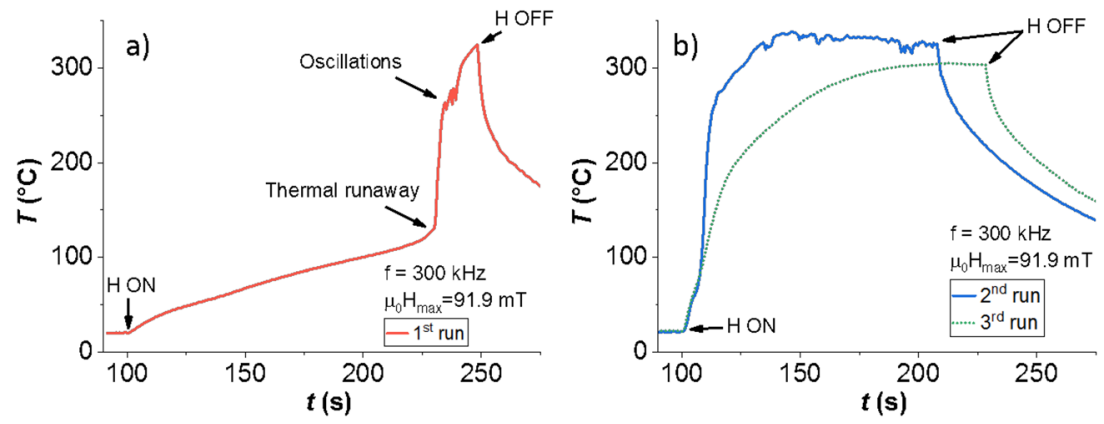

FIG. 9. Raw temperature measurements of Co-NRs on Siralox-5( with an applied magnetic field intensity of $\mu_{0} H_{\max }$ $=91.9 \mathrm{mT}$ at a frequency $f=300 \mathrm{kHz}$ : (a) First experiment run on a fresh sample. (b) Second and third measurements performed just after the first one. 

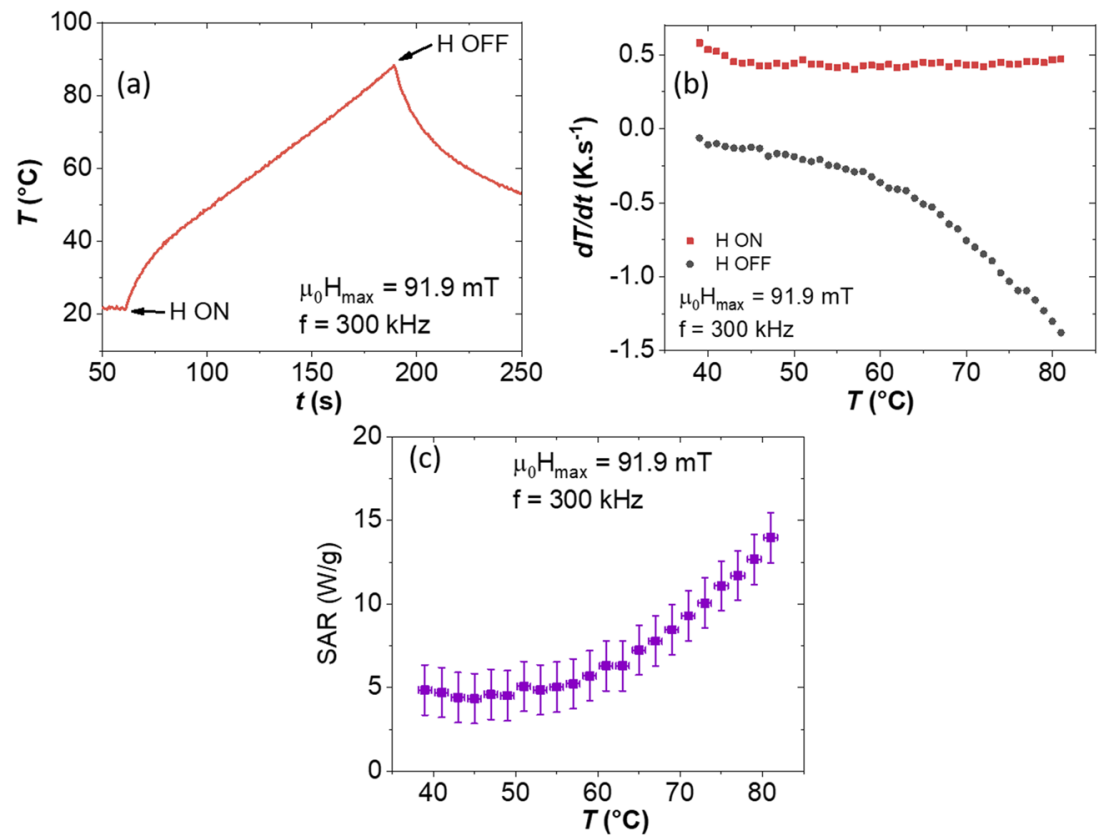

reforming reactions. This is the main subject of another publication in which the detailed SAR(T) of the samples can be found. ${ }^{14}$ The measurements were performed under argon at ambient pressure in both calorimeter chambers. From the data shown in that work, the coefficient of SAR variation can be inferred. For $x=0.5$, the variation of SAR is $\triangle S A R / \triangle T=-0.76 \mathrm{~W} \mathrm{~g}^{-1} \mathrm{~K}^{-1}$ or $-0.4 \% \mathrm{~K}^{-1}$. For $x=0.8$, the variation of SAR is $\Delta S A R / \Delta T=-4.2 \mathrm{~W} \mathrm{~g}^{-1} \mathrm{~K}^{-1}$ or $-0.29 \% \mathrm{~K}^{-1}$.

We will here focus on the fact that, in some samples, the NPs form chains, which has a strong influence on the measurement results. The sample of interest here is the sample $x=0.8$. As shown in our previously published paper, ${ }^{14}$ in this sample, the NPs form chains in spite of being supported on Siralox-5๑. We have been able to measure the temperature of the sample and move the measurement point once the equilibrium temperature was reached. We moved it from a point on the sample without chains to a point where we observed chains (which was possible using the laser integrated to the pyrometer since chains were visible to the naked eye). This displacement was of $\sim 2 \mathrm{~mm}$. The results are shown in Fig. 11, where (b) is only a zoomed-in view of (a) in the region where the measurement point has been moved.
The first observation is that the maximum temperature reached is $T=549^{\circ} \mathrm{C}$, which shows the capability of the setup to measure high temperatures without any problem. The second observation is that, when we moved the measurement point, the temperature increased from $T=446{ }^{\circ} \mathrm{C}$ to $T=549^{\circ} \mathrm{C}$. This is an additional experimental proof that the formation of chains within an assembly of MNPs leads to a strong increase in their heating power. This behavior has been shown previously by other groups and ours by studying different samples. However, using this pyrometer, we were able to show that, within the same sample, regions with chains are much hotter than regions without, inducing temperature gradients, which are in our case larger than $100^{\circ} \mathrm{C}$.

\section{CONCLUSION}

We report in this paper a method and a setup for the measurement of NP heating power and the temperature variation of this heating power. It is expected that the setup works up to $900^{\circ} \mathrm{C}$ as it is the detection limit of the pyrometer, here we present measurements up to $549^{\circ} \mathrm{C}$, under vacuum or under a controlled atmosphere. We have described in detail the setup, its ability, and limitations.

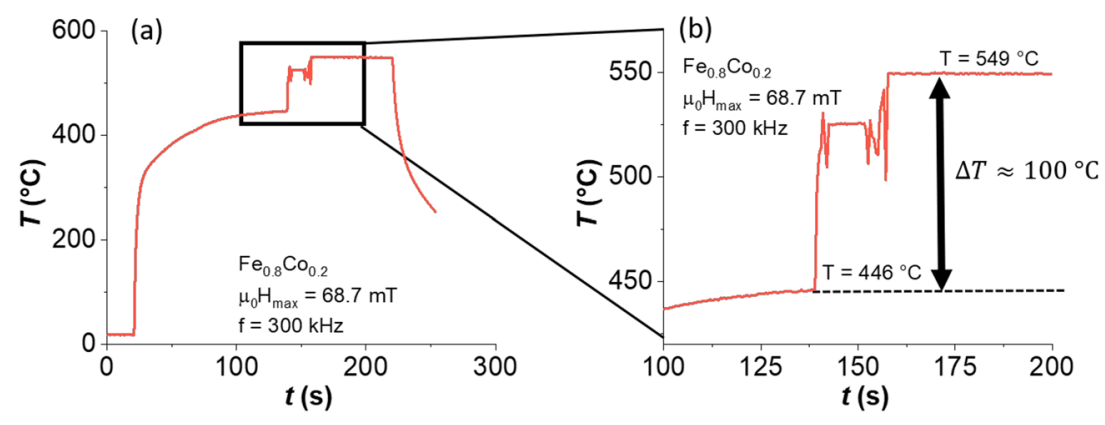

FIG. 11. (a) Temperature measurements of the $\mathrm{Fe}_{0.8} \mathrm{Co}_{0.2}$ sample with a displacement of the measurement spot. (b) Zoomed-in view of the same curve on the temperature difference due to the spot displacement. 
The setup versatility was checked with the measurements of highly disparate samples. We have shown that the setup permits to measure, as expected, both non-magnetic (NEG sample, displaying a weakly temperature-dependent SAR) and magnetic materials. Three different nanostructured magnetic materials have been assessed. Soft magnetic $\mathrm{Fe}_{2} \mathrm{O}_{3} @ \mathrm{SiO}_{2}$ NPs show a decrease in SAR with temperature, whereas hard magnetic cobalt NRs show an increase in SAR with temperature. Both behaviors were attributed to the same origin: the decrease in coercivity with temperature. Abrupt increases in temperature in the Co nanorod samples were also observed and attributed to the irreversible structural evolution of the sample. Importantly, our setup is suitable to detect variations in physical properties such as the coercivity and the thermal stability, which are key factors for a proper use of magnetic NPs in magnetically heated catalysis reactions. Our last experiment on FeCo MNPs shows that strong temperature gradients can arise in the calorimeter due to the presence of chains, leading to temperature gradients within the sample and non-treatable data. In addition, it is important for us to say that the setup and method are easily implementable in other laboratories as its cost is limited, and the method is fully described. Finally, it is expected that this setup will allow monitoring a magnetically heated catalytic process (work currently under process) by replacing the calorimeter by a catalytic reactor and by regulating the temperature with a PID (proportional integral derivative) loop. Notably, this new type of measurement will offer a better control of the temperature of the chemical reaction, leading to improved chemical yields and less power consumption.

\section{ACKNOWLEDGMENTS}

The authors acknowledge the ERC Advanced Grant (No. MONACAT 2015-694159). M.E. acknowledges the European Union's Horizon 2020 research and innovation programme (Marie Skłodowska-Curie Grant Agreement No. 704098) for the execution of the project related to this article and the Spanish MICINN for the current Ramón y Cajal Contract (No. RYC2018-024396-I). D.Y. acknowledges the financial support of the "CaSh" project through EUR Grant NanoX No. ANR-17-EURE-0009 in the framework of the "Programme des Investissements d'Avenir."

\section{DATA AVAILABILITY}

The data that support the findings of this study are available from the corresponding author upon reasonable request.

\section{REFERENCES}

${ }^{1}$ E. A. Périgo, G. Hemery, O. Sandre, D. Ortega, E. Garaio, F. Plazaola, and F. J. Teran, "Fundamentals and advances in magnetic hyperthermia," Appl. Phys. Rev. 2(4), 041302 (2015).

${ }^{2}$ S. Ceylan, C. Friese, C. Lammel, K. Mazac, and A. Kirschning, "Inductive heating for organic synthesis by using functionalized magnetic nanoparticles inside microreactors," Angew. Chem., Int. Ed. 47(46), 8950-8953 (2008).

${ }^{3} \mathrm{Y}$. Schaafsma, "Improvements in or relating to supplying heat to fluids," GB925025A, May 1, 1963.

${ }^{4}$ C. Friese, A. Kirschning, J. Wichelhaus, and S. V. Ceylan, "Method for carrying out chemical reactions with the aid of an inductively heated heating medium," International patent $\mathrm{WO} / 2009 / 074373$ (19 June 2009).

${ }^{5}$ S. Ceylan, L. Coutable, J. Wegner, and A. Kirschning, "Inductive heating with magnetic materials inside flow reactors,” Chem.-Eur. J. 17(6), 1884-1893 (2011).
${ }^{6}$ J. Hartwig, S. Ceylan, L. Kupracz, L. Coutable, and A. Kirschning, "Heating under high-frequency inductive conditions: Application to the continuous synthesis of the neurolepticum olanzapine (Zyprexa)," Angew. Chem., Int. Ed. 52(37), 9813-9817 (2013).

${ }^{7}$ A. Meffre, B. Mehdaoui, V. Connord, J. Carrey, P. F. Fazzini, S. Lachaize, M. Respaud, and B. Chaudret, "Complex nano-objects displaying both magnetic and catalytic properties: A proof of concept for magnetically induced heterogeneous catalysis," Nano Lett. 15(5), 3241-3248 (2015).

${ }^{8}$ A. Bordet, L.-M. Lacroix, P.-F. Fazzini, J. Carrey, K. Soulantica, and B. Chaudret, "Magnetically induced continuous $\mathrm{CO}_{2}$ hydrogenation using composite iron carbide nanoparticles of exceptionally high heating power," Angew. Chem., Int. Ed. 55(51), 15894-15898 (2016)

${ }^{9}$ Y. Liu, N. Cherkasov, P. Gao, J. Fernández, M. R. Lees, and E. V. Rebrov, "The enhancement of direct amide synthesis reaction rate over $\mathrm{TiO}_{2} @ \mathrm{SiO}_{2} @ \mathrm{NiFe}_{2} \mathrm{O}_{4}$ magnetic catalysts in the continuous flow under radiofrequency heating," J. Catal. 355, 120-130 (2017).

${ }^{10}$ P. M. Mortensen, J. S. Engbæk, S. B. Vendelbo, M. F. Hansen, and M. Østberg, "Direct hysteresis heating of catalytically active $\mathrm{Ni}-\mathrm{Co}$ nanoparticles as steam reforming catalyst," Ind. Eng. Chem. Res. 56(47), 14006-14013 (2017).

${ }^{11}$ M. G. Vinum, M. R. Almind, J. S. Engbæk, S. B. Vendelbo, M. F. Hansen, C. Frandsen, J. Bendix, and P. M. Mortensen, "Dual-function cobalt-nickel nanoparticles tailored for high-temperature induction-heated steam methane reforming," Angew. Chem., Int. Ed. 57(33), 10569-10573 (2018).

${ }^{12}$ W. Wang, C. Duong-Viet, Z. Xu, H. Ba, G. Tuci, G. Giambastiani, Y. Liu, T. Truong-Huu, J.-M. Nhut, and C. Pham-Huu, " $\mathrm{CO}_{2}$ methanation under dynamic operational mode using nickel nanoparticles decorated carbon felt (Ni/OCF) combined with inductive heating," Catal. Today 357, 214 (2019).

${ }^{13}$ W. Wang, G. Tuci, C. Duong-Viet, Y. Liu, A. Rossin, L. Luconi, J.-M. Nhut, L. Nguyen-Dinh, C. Pham-Huu, and G. Giambastiani, "Induction heating: An enabling technology for the heat management in catalytic processes," ACS Catal. 9, 7921-7935 (2019).

${ }^{14}$ J. Marbaix, N. Mille, L.-M. Lacroix, J. M. Asensio, P.-F. Fazzini, K. Soulantica, J. Carrey, and B. Chaudret, "Tuning the composition of FeCo nanoparticle heating agents for magnetically induced catalysis," ACS Appl. Nano Mater. 3(4), 3767-3778 (2020).

${ }^{15}$ M. Veverka, P. Veverka, O. Kaman, A. Lančok, K. Závěta, E. Pollert, K. Knížek, J. Boháček, M. Beneš, P. Kašpar, E. Duguet, and S. Vasseur, "Magnetic heating by cobalt ferrite nanoparticles," Nanotechnology 18(34), 345704 (2007).

${ }^{16}$ E. Garaio, O. Sandre, J.-M. Collantes, J. A. Garcia, S. Mornet, and F. Plazaola, "Specific absorption rate dependence on temperature in magnetic field hyperthermia measured by dynamic hysteresis losses (ac magnetometry)," Nanotechnology 26(1), 015704 (2014).

${ }^{17}$ E. Natividad, M. Castro, and A. Mediano, "Adiabatic magnetothermia makes possible the study of the temperature dependence of the heat dissipated by magnetic nanoparticles under alternating magnetic fields," Appl. Phys. Lett. 98(24), 243119 (2011).

${ }^{18} \mathrm{E}$. Natividad and I. Andreu, "Omitting the need of external heat capacity data in an adiabatic magnetothermal setup devoted to the characterization of nanomaterials for magnetic hyperthermia," Appl. Therm. Eng. 117, 409-416 (2017).

${ }^{19}$ M. Coïsson, G. Barrera, C. Appino, F. Celegato, L. Martino, A. P. Safronov, G. V. Kurlyandskaya, and P. Tiberto, "Specific loss power measurements by calorimetric and thermal methods on $\gamma$ - $\mathrm{Fe}_{2} \mathrm{O}_{3}$ nanoparticles for magnetic hyperthermia," J. Magn. Magn. Mater. 473, 403-409 (2018).

${ }^{20}$ F. Varsano, M. Bellusci, A. La Barbera, M. Petrecca, M. Albino, and C. Sangregorio, "Dry reforming of methane powered by magnetic induction," Int. J. Hydrogen Energy 44, 21037 (2019).

${ }^{21}$ P. Uprety, M. M. Junda, and N. J. Podraza, "Optical properties of borosilicate glass from $3.1 \mathrm{~mm}$ to $210 \mathrm{~nm}(0.4 \mathrm{meV}$ to $5.89 \mathrm{eV})$ by spectroscopic ellipsometry," Surf. Sci. Spectra 24(2), 026003 (2017).

${ }^{22} \mathrm{M}$. He, W. Yan, Y. Chang, K. Liu, and X. Liu, "Fundamental infrared absorption features of $\alpha$-quartz: An unpolarized single-crystal absorption infrared spectroscopic study," Vib. Spectrosc. 101, 52-63 (2019).

${ }^{23}$ See https://numpy.org/doc/stable/reference/generated/numpy.gradient.html for numpy.gradient-NumPy v1.20 manual; accessed March 11, 2021. 
${ }^{24}$ See https://www.advancedenergy.com/products/temperature-measurement/ thermal-measurement-optical-pyrometers-power-controllers/non-metal-applica tions-pyrometers/in-5-in-5-plus/ for Impac IN 5 and IN 5 Plus Series|Pyrometers| Advanced Energy; accessed March 11, 2021.

${ }^{25}$ Transmetra Company, Table of Emissivity of Various Surfaces.

${ }^{26}$ See https://www.bipm.org/en/publications/guides/gum.html for BIPM-Guide to the Expression of Uncertainty in Measurement (GUM); accessed March 11, 2021.

${ }^{27}$ Y. S. Touloukian and E. H. Buyco, Thermophysical Properties of Matter, The TPRC Data Series Vol. 4, Specific Heat: Metallic Elements and Alloys (Thermophysical and Electronic Properties Information Analysis Center, Lafayette, IN, 1971).

${ }^{28}$ Y. S. Touloukian and E. H. Buyco, Thermophysical Properties of Matter, The TPRC Data Series Vol. 5, Specific Heat: Nonmetallic Solids (Thermophysical and Electronic Properties Information Analysis Center, Lafayette, IN, 1970).

${ }^{29} \mathrm{R}$. Taherian, "7-Application of polymer-based composites: Bipolar plate of PEM fuel cells," in Electrical Conductivity in Polymer-Based Composites, edited by R. Taherian and A. Kausar (Plastics Design Library; William Andrew Publishing, 2019), pp. 183-237.

${ }^{30}$ A. T. D. Butland and R. J. Maddison, "The specific heat of graphite: An evaluation of measurements," J. Nucl. Mater. 49(1), 45-56 (1973).

${ }^{31}$ F. Fiorillo, Measurement and characterization of magnetic materials, https:// cds.cern.ch/record/887174; accessed March 11, 2021.

${ }^{32}$ E. J. Davies, Conduction and Induction Heating (IET Digital Library, 1990).
${ }^{33} \mathrm{R}$. Massart, "Preparation of aqueous magnetic liquids in alkaline and acidic media," IEEE Trans. Magn. 17(2), 1247-1248 (1981).

${ }^{34}$ J. Carrey, B. Mehdaoui, and M. Respaud, "Simple models for dynamic hysteresis loop calculations of magnetic single-domain nanoparticles: Application to magnetic hyperthermia optimization," J. Appl. Phys. 109(8), 083921 (2011).

${ }^{35}$ B. Cormary, T. Li, N. Liakakos, L. Peres, P.-F. Fazzini, T. Blon, M. Respaud, A. J. Kropf, B. Chaudret, J. T. Miller, E. A. Mader, and K. Soulantica, "Concerted growth and ordering of cobalt nanorod arrays as revealed by tandem in situ SAXSXAS studies," J. Am. Chem. Soc. 138(27), 8422-8431 (2016).

${ }^{36}$ S. S. Kale, J. M. Asensio, M. Estrader, M. Werner, A. Bordet, D. Yi, J. Marbaix, P.-F. Fazzini, K. Soulantica, and B. Chaudret, "Iron carbide or iron carbide/cobalt nanoparticles for magnetically-induced $\mathrm{CO}_{2}$ hydrogenation over $\mathrm{Ni} / \mathrm{SiRAlOx}$ catalysts," Catal. Sci. Technol. 9(10), 2601-2607 (2019).

${ }^{37}$ S. Lentijo-Mozo, R. P. Tan, C. Garcia-Marcelot, T. Altantzis, P.-F. Fazzini, T. Hungria, B. Cormary, J. R. Gallagher, J. T. Miller, H. Martinez, S. Schrittwieser, J. Schotter, M. Respaud, S. Bals, G. V. Tendeloo, C. Gatel, and K. Soulantica, "Air- and water-resistant noble metal coated ferromagnetic cobalt nanorods," ACS Nano 9(3), 2792-2804 (2015).

${ }^{38} \mathrm{~W}$. J. Carr, "Temperature dependence of ferromagnetic anisotropy," Phys. Rev. 109(6), 1971-1976 (1958)

${ }^{39}$ M. Takahashi, S. Kadowaki, T. Wakiyama, T. Anayama, and M. Takahashi, "Magnetocrystalline anisotropy of Co and Co-Ni alloys," J. Phys. Soc. Jpn. 44(3), 825-832 (1978). 